Beitrag in einem Sammelband

\title{
Das metabolische Syndrom im Alltag - translation im Zeitalter von Biosozialität
}

\author{
Jörg Niewöhner*
}

2008

Dies ist die akzeptierte Manuskriptversion (Postprint) des folgendermaßen veröffentlichten Sammelbandbeitrags:

Titel

Autor

Veröffentlichungsdatum

Titel des Sammelbands

Herausgeber des Sammelbands

Seiten

Verlag

DOI
Das metabolische Syndrom im Alltag - translation im Zeitalter von Biosozialität

Niewöhner, Jörg

2008

Kreuzzug gegen Fette: Sozialwissenschaftliche Aspekte des gesellschaftlichen Umgangs mit Übergewicht und Adipositas

Schmidt-Semisch, Henning; Schorb, Friedrich 191-206

VS Verlag für Sozialwissenschaften / Springer 10.1007/978-3-531-90800-7_12 


\title{
Das metabolische Syndrom im Alltag - translation im Zeitalter von Biosozialität
}

\author{
Jörg Niewöhner
}

2008

\section{Einleitung}

Das Konzept der Biosozialität (Rabinow 1992) verweist auf eine mannigfaltige Verschränkung verschiedener Phänomene, die gemeinhin als entweder natürlich oder kulturell verstanden werden, und damit zugleich auf eine zunehmende gegenseitige Durchdringung medizinischer und sozialer Praxen. Besonders augenscheinlich wird diese Verschränkung an den Schnittstellen zwischen einerseits hochkomplexen Expertensystemen, wie Wissenschaft, Medizin und Technologieentwicklung, und andererseits hochgradig ausdifferenzierten sozialen Alltagen 1 Vor allem in der angloamerikanischen Wissenschaftsforschung und der kritischen Medizinanthropologie wird derweil versucht, diese gegenseitige Durchdringung nicht als interface (im Sinne eines Berührungspunktes) zweier voneinander getrennter Entitäten bzw. Diskurse zu begreifen, sondern stattdessen mit dem Konzept der intersections (Lock et al 2000) bzw. der translation (Callon 1999) die interaktive Dynamik verschiedener Wissenspraxen zu thematisieren. Diese Herangehensweise rekurriert vornehmlich auf Ansätze, die die alltäglichen Aushandlungen zwischen Akteuren, Wissen und Artefakten in den Vordergrund rücken. Sie gelangt dadurch zu einem Verständnis konkreter sozialer Konstellationen, die sich dadurch auszeichnen, dass Entitäten und Phänomene gleichzeitig anwesend und miteinander auf vielfältige Art und Weise verschränkt sind. Dort wo die analytische Trennung von ExpertInnen und PatientInnen ein interface, also eine Grenze und damit Transfer thematisiert, betont der Begriff der intersections die Reproduktion von Praxen.

Das Konzept der translation baut auf dieser Perspektive auf und verbindet sie mit Elementen einer Actor-Network-Theory (ANT: Latour 2005; Law 1992; Law /Hassard 1999). Dabei bezeichnet translation gerade nicht Übersetzung im Sinne einer einfachen

\footnotetext{
${ }^{1}$ Diese Schnittstellen sind vielfach untersucht worden: in Analysen von medialer Vermittlung, in den Defizitmodellen verschiedener Rezeptionstheorien oder im empowerment des Public Understanding of Science and Humanities (Stifterverband 2000). Die problematischen Konzeptionen von sozialem Alltag und von Transferprozessen, die diesen Ansätzen implizit zugrunde liegen, sind bereits umfassend kritisiert worden (Irwin 2001; Wynne 1996).
} 
Übertragung, sondern lässt sich besser aus seinen romanischen Wurzeln heraus mit Verschiebung übersetzen. Mit der Einbettung in ANT rücken zwei Dinge in den Vordergrund: Zum einen wird nicht-menschlichen Akteuren Handlungsträgerschaft zugestanden, d. h. Artefakte und Technologien sind aktiv in translationen eingebunden. Zum anderen wird auf dieser Basis Alltag als sich reproduzierende Praxis, als sozio-technisches Netzwerk verstanden, das permanent in Bewegung ist und sich verschiebt. Diese Verschiebungen bezeichnet das Konzept der translation. Es rückt damit ab von akteurszentrierten und intentionalen Handlungskonzepten der Soziologie, die Wissenstransfer als diskursiv oder kognitiv begreifen, und bettet Wissen als Praxis in dynamische sozio-technische Netzwerke ein.2

Vor diesem theoretischen Hintergrund und am Beispiel einer Analyse von Übergewicht als präventive Praxis versucht der vorliegende Beitrag, eine doppelt symmetrische Perspektive zu entwickeln: Zum einen stehen medizinisches Experten- und alltägliches Laienwissen als interagierende Wissenspraxen gleichberechtigt nebeneinander (Bloor 1991). Zum anderen unterscheidet die Analyse nicht kategorisch zwischen Materialitäten und Diskursen im Hinblick auf ihre Fähigkeiten, Praxis zu verändern (Latour 1995). In den Vordergrund rückt zum einen die Frage, wie unterschiedliche medizinische Praxen ein panoply of pastoral keeper $\$^{3}$ formen bzw. verändern. Zum anderen werden die Effekte dieses panoply als Klassifizierungseffekte thematisiert. Ian Hacking spricht in diesem Zusammenhang von making up people, um zu problematisieren, dass Klassifizierungen immer auch Reaktionen der Klassifizierten herausfordern (Hacking 1999; 2006). Aufgrund seiner wissenschaftsphilosophischen Orientierung auf Fragen des dynamischen Nominalismus steht dabei meist eine individualistische Perspektive im Vordergrund. In diesem Beitrag hingegen wird diese Sicht um eine Betrachtung von Kollektivierungseffekten und Widerständigkeiten erweitert.

Die folgenden Abschnitte entwickeln zwei Fallbeispiele - Ethnizität in der Diagnostik und Size Acceptance - anhand derer zum einen deutlich wird, was das Konzept der translation sichtbar machen kann. Zum anderen versucht die Diskussion dieser Perspektive zu zeigen, dass eine solche Analyse komplementär zu einem weiter verbreiteten Foucaultschen Ansatz agieren kann und sollte. Sie stellt sich in keiner Weise gegen die Thematisierung von Subjektivierungs- und Medikalisierungsprozessen, sondern ändert lediglich den Fokus der empirischen Betrachtung weg von Diskursen bzw. weg von einem dispositif und hin zu einer Betrachtung von Praxis in sozio-technischen Netzwerken. Sie erarbeitet sich damit eine Kritikfähigkeit, die in Richtung naturwissenschaftlich-medizinischer Praxis eine andere Anschlussfähigkeit ermöglicht.

\footnotetext{
${ }^{2}$ Diese notwendige Verkürzung tut einigen Ansätzen gerade aus dem Bereich der Techniksoziologie unrecht (siehe daher Bijker et al 1987; Joerges 1987; 1996).

${ }^{3}$ Panoply of pastoral keepers bezeichnet hier die Mannigfaltigkeit und die Verschränkung der Hüter pastoraler Macht, d.h. der Diskurse der Biomacht, die durch Subjektivierung und Technologien des Selbst regieren. Foucault ordnet diese Macht Diskursen bzw. dispositifs zu und nicht Akteuren oder Institutionen.
} 


\section{Ethnizität in der Diagnostik}

Übergewicht wird seit Mitte der 1980er Jahre vor allem in der Forschung im Zusammenhang des metabolischen Syndroms thematisiert. Es rückt damit aus einer engen Konzeptionalisierung von energetischer Balance in eine komplexe Sicht von Stoffwechselveränderungen, deren Ursachen und Dynamik erst rudimentär verstanden sind. Das metabolische Syndrom als nosologische Einheit verweist auf die vor allem aus epidemiologischen Studien hervorgegangene Beobachtung, dass bestimmte metabolische Veränderungen häufig gemeinsam auftreten und damit das Risiko von Herzkreislauferkrankungen signifikant erhöhen (z. B.:Zimmet et al 2005). Als Indikatoren dieser metabolischen Veränderungen werden im allgemeinen Übergewicht, ungünstige Fettstoffwechselveränderungen (Cholesterin, Triglyzeride), Bluthochdruck und erhöhte nüchterne Glukosewerte diskutiert (NCEP 2001). Ferner wächst die Gewissheit, dass das Hormonsystem über verschiedene Wege involviert ist (Brunner et al 2002). Als Folgen einer ungünstigen Bündelung dieser Faktoren scheinen Diabetes mellitus und langfristig vor allem Arteriosklerose und Herzinfarkt gehäuft aufzutreten. Hinzu kommen mehr oder weniger intensiv diskutierte Hypothesen von Schlafstörungen bis zu Krebserkrankungen (Pasanisi et al 2006; Tauman et al 2005). Als eine der wichtigsten proximalen Ursachen wird z. Z. die Insulinresistenz gehandelt, die sowohl genetisch als auch durch Umweltfaktoren bedingt zu sein scheint (Reaven 2002). Als distale Ursachen rücken damit vor allem spezifische Mutationen, Lebensstilfaktoren und frühkindliche Prägungseffekte in das Visier der ForscherInnen (Armitage et al 2004).

Seit seiner Konzeption Mitte der 1980er Jahre jedoch sind die Existenz dieses Syndroms, seine Aussagekraft als Risikomarker über konventionellere Modelle hinaus, seine Ätiologie und seine Diagnose heftig umstritten (Kahn et al 2005; Reaven 1988). Dabei verlaufen die Störungszonen vor allem entlang disziplinärer Prägungen und Institutionalisierungen, mit denen zum einen unterschiedliche methodische Zugänge, zum anderen aber auch divergierende Absichten verknüpft sind $\mathrm{d}^{4}$ Es ist nicht die Absicht der folgenden Abschnitte, diese Kontroverse aufzuarbeiten. Vielmehr geht es um Ausschnitte aus einem intensiv beforschten Feld, die für die intersections zwischen Expertensystemen und sozialen Alltagen von besonderer Relevanz sind.

Neben ätiologischen Diskussionen über das metabolische Syndrom ist auch seine Diagnose heftig umstritten (Cheal et al 2004). Welche Parameter herangezogen werden sollen, in welcher Kombination und mit welchen Grenzwerten, wird immer wieder von verschiedenen Fachgesellschaften revidiert. Laut der Definition von 2005 der International Diabetes Federation (IDF 2006) muss für eine vollständige Diagnose in jedem Fall Übergewicht vorliegen und zwei von vier weiteren Faktoren (Triglyzeride, Cholesterin, Blutdruck, nüchtern Glukose). Übergewicht wird in den meisten Fällen über den so genannten body-mass-index erfasst $\left(\mathrm{kgm}^{-2}\right)$, der eine Größe-Gewicht Relation abbildet. Neuere Befunde zur hormonellen Wirksamkeit bestimmter Fettzellen legen z. Z. allerdings nahe, dass gerade das viszerale Fett, d. h. das Fett im Bauchraum, für die kardiovaskulären

${ }^{4}$ Sowohl von Chris Chatterton, Cardiff University und CESAGEN, als auch von Martin Döring, Universität Hamburg, BIOGUM, sind Publikationen zu dieser Problematik in Arbeit. 
Folgeerscheinungen des Übergewichts verantwortlich sein könnte (Rosmond/Bjorntorp 2000). Die IDF hat daher den Bauchumfang statt des BMI in seinen Diagnosekatalog aufgenommen. Um die Spezifität dieses Parameters zu erhöhen, werden die Grenzwerte für diesen Umfang ethnisch stratifiziert (siehe Abb. 1 und http://www.idf.org/).

\begin{tabular}{|c|c|c|}
\hline \multicolumn{2}{|l|}{ Country/Ethnic group } & \multirow{2}{*}{$\begin{array}{l}\text { Waist circumference }^{*} \\
\geq 94 \mathrm{~cm}\end{array}$} \\
\hline Europids & Male & \\
\hline $\begin{array}{l}\text { In the USA, the ATP III } \\
\text { values (102 cm male; } 88 \mathrm{~cm} \\
\text { female) are likely to continue } \\
\text { to be used for clinical } \\
\text { purposes }\end{array}$ & Female & $\geq 80 \mathrm{~cm}$ \\
\hline \multirow{2}{*}{$\begin{array}{l}\text { South Asians } \\
\text { Based on a Chinese, Malay } \\
\text { and Asian-Indian population }\end{array}$} & Male & $\geq 90 \mathrm{~cm}$ \\
\hline & Female & $\geq 80 \mathrm{~cm}$ \\
\hline \multirow[t]{2}{*}{ Chinese } & Male & $\geq 90 \mathrm{~cm}$ \\
\hline & Female & $\geq 80 \mathrm{~cm}$ \\
\hline \multirow[t]{2}{*}{ Japanese } & Male & $\geq 85 \mathrm{~cm}$ \\
\hline & Female & $\geq 90 \mathrm{~cm}$ \\
\hline $\begin{array}{l}\text { Ethnic South and Central } \\
\text { Americans }\end{array}$ & \multicolumn{2}{|c|}{$\begin{array}{l}\text { Use South Asian recommendations until more specific } \\
\text { data are available }\end{array}$} \\
\hline Sub-Saharan Africans & \multicolumn{2}{|c|}{ Use European data until more specific data are available } \\
\hline $\begin{array}{l}\text { Eastern Mediterranean } \\
\text { and Middle East (Arab) } \\
\text { populations }\end{array}$ & \multicolumn{2}{|c|}{ Use European data until more specific data are available } \\
\hline
\end{tabular}

Abbildung 1: Ethnisch spezifizierter Bauchumfang bei der Diagnose des metabolischen Syndroms (IDF 2005)

Die Erläuterungen der IDF weisen ausdrücklich daraufhin, dass es sich hier um ethnische Zugehörigkeit und nicht um Aufenthaltsland („country of residence“) handelt. Zwar stammen die Daten für eine solche Stratifizierung aus epidemiologischen und nicht aus genetischen Daten. Trotzdem wird mit dem Terminus „ethnic group“ auf eine angeblich biologisch homogene Gruppe verwiesen. Unabhängig davon, ob eine derart differenzierende Diagnostik im klinischen Alltag brauchbare Ergebnisse produziert, verweist sie auf eine ganze Reihe problematischer Diskussionen (Duster 2006). Die Existenz von Menschengruppen, die im Hinblick auf komplexe Krankheitsbilder wie das metabolische Syndrom biologisch homogen genug sind, um von klinischer Relevanz zu sein, wird in den allermeisten wissenschaftlichen Zirkeln heftig bestritten. Die genetische Varianz zwischen Gruppen erscheint in den meisten Studien geringer als die Varianz innerhalb solcher Gruppen (AAA 1998). Nichtsdestotrotz finden sich solche Gruppen als Rasse oder Ethnie in vielen aktuellen Forschungspraxen, ohne problematisiert zu werden (Lipphardt/Niewöhner 2007). Und auch in dieser Leitlinie verweist das Konzept der Europids auf genetisch determinierte Unterschiede. Dabei ist weitgehend unklar, wie genau ein erhöhtes Herzkreislaufrisiko zustande kommt und damit auch wer innerhalb einer solchen 
nur scheinbar homogenen Gruppe ein erhöhtes Risiko aufweist. Dass manche Menschen Fett anders verstoffwechseln als andere, dass also biologische Unterschiede bestehen, ist dabei unbestritten. Die Frage ist vielmehr, ob Ethnie einen vertretbaren Zugang zu dieser Gruppe darstellt.

Es wird deutlich, dass das Konzept der Population und damit der populationsbezogenen Grenzwerte immer schon die Manifestation vielfältiger Wissenspraxen darstellt. Diese in den Blick zu nehmen, ist von zentraler Bedeutung. Denn ein Verständnis der translationen, die Epidemiologie in Bezug setzen zu viszeralem Fett, zu genetischer Prädisposition von Insulinresistenz, zu Herzkreislaufrisiko, ein solches Verständnis zeigt gewissermaßen die Mechanik hinter dem Ergebnis oder der Fassade, an der häufig angesetzt wird, um die Medikalisierung sozialer Alltage zu kritisieren.

Die Implementierung der besagten Grenzwerte verläuft z.Z., nicht zuletzt wegen der umstrittenen Wissensbasis, nicht einheitlich. Während IDF und die International Obesity Task Force (IOTF, eine Arbeitsgruppe der International Association for the Study of Obesity, IASO) weiter an ethnisch stratifizierten Grenzwerten arbeiten, hat sich diese Perspektive weder in nationalen Verbänden in Deutschland noch auf der Ebene der World Health Organisation bisher vollständig durchgesetzt (WHO Expert Consultation 2004). Die WHO empfiehlt auf der Basis epidemiologischer Daten, dass Interventionen den Situationen der jeweiligen Länder angepasst werden sollten. Dies hat dazu geführt, dass manche Länder, wie z. B. Singapur, aufgrund der Evidenz für asiatische Populationen die strikteren Grenzwerte in ihre nationalen public health Strategien übernehmen, andere hingegen nicht.

Von Interesse in diesem Kontext ist die Reaktion betroffener „Risikogruppen“. Die jährliche Konvention der National Association for the Advancement of Colored People (NAACP) wurde 2006 von Bruce Gordon eröffnet: „... We've got a Freedom Fighters Fitness Challenge. Go to the workshops, check it out. It speaks to obesity, and it speaks to the fact that there is a higher percentage of obesity in our communities than there is in the majority community. And we know all of the bad things that go along with obesity, like diabetes, and high blood pressure, and heart disease. And we know that we've got it, right." (Bruce S. Gordon addresses the 97th NAACP Convention, 2006) Noch deutlicher wird die Ansage in der Health Watch Sektion des San Jose Chapters derselben Organisation: „With genetic predispositions coupled with poor diet and little to no physical activity, these numbers will only increase."

Diese Form der positiven Aneignung stellt keinesfalls die Regel dar, wie die zweite Fallstudie zeigt. Sie verdeutlicht jedoch, wie translationen ablaufen. Eine heterogene Wissenspraxis, nämlich eine differenzierte Diagnostik für Übergewicht im Hinblick auf kardiovaskuläre Risiken, materialisiert sich in Form von Grenzwerten. Sie verliert dadurch einen Großteil ihrer Kontingenz und wird zu einer Art standardised package (Fujimura 1992), das jetzt in den USA colored people mit genetischen Prädispositionen, mit Risikoprofilen und letztlich auch mit neuen Therapien in Verbindung bringt. Es verschiebt sich also die Art und Weise, wie eine community sich selbst weiss und versteht. Diese veränderten Wissenspraxen lassen zwar Widerstand zu, verweisen aber nicht mehr auf die Kontingenz der translation, d. h. eine wissenschaftliche Kontroverse wird über veränderte 
Wissenspraxen und ihre Materialisierungen unsichtbar.5

\section{Genetik und Size Acceptance}

Die vorangegangenen Abschnitte haben die mannigfaltigen wissenschaftlichen Unsicherheiten verdeutlicht, die nach wie vor in Bezug auf die Ursachen kardiovaskulärer Erkrankungen bestehen. Zwar scheint die epidemiologische Evidenz erdrückend, dass Übergewicht das Risiko einer Herzkreislauferkrankung erhöht. Diese Daten erklären jedoch weder Kausalbeziehungen, sondern lediglich Korrelationen, noch sind sie in der Lage, Aussagen auf der Ebene von Einzelpersonen zu treffen. Die Forschungsanstrengungen zielen daher vermehrt auf die Identifikation von Biomarkern, die spezifischere Risikoprofile und vor allem gezieltere therapeutische Ratschläge ermöglichen. Dies gestaltet sich jedoch nicht zuletzt deshalb schwierig, da die Forschung das Netzwerk möglicher Ursachen immer weiter aufspannt und ausdifferenziert. Was zunächst lediglich als das Problem einer positiven Energiebilanz diskutiert wurde, verschränkt heute genetische Prädispositionen, Entzündungs- und Stoffwechselparameter, neuroendokrine Faktoren, Lebensstile und Umweltfaktoren. Diese zunehmende Komplexität legt den Schluss nahe, dass die nächsten Jahre von mehr oder weniger aggregierten Risikoprofilen bestimmt sein werden, die auf der Basis von Kombinationen molekularer, physiologischer, psychologischer, sozialer und Umweltfaktoren operieren werden. Individuelle Diagnostik, Vorbeugung und Therapie scheinen in weiter Ferne (Hedgecoe 2004; Kollek et al. 2003).

Dabei geht der Druck, wirksame Vorbeugung mittels Profilbildung und Prävention zu entwickeln und anzuwenden, zunehmend weniger von der Medizin selbst aus, als von einer breiten Allianz aus Politik, Wirtschaft und Wissenschaft, die sich um die Zukunft der Kranken- und Rentenversicherungen bzw. des Sozialstaats insgesamt sorgt (Walter/Scriba 2004). Die zunehmende Prävalenz von Übergewicht und seiner chronischen Folgeerkrankungen, führt zum einen dazu, dass mehr Menschen teure Gesundheitsleistungen in Anspruch nehmen; zum anderen scheiden die gleichen Menschen früher aus dem Erwerbsleben aus und entfallen als Einzahler in diverse Sicherungssysteme. Um diese Entwicklung zu stoppen und umzukehren, werden Präventionsbestrebungen aller Art massiv gefördert ${ }^{6}$ Neu ist dabei in Deutschland vor allen Dingen, dass man beginnt, deutlich über bloße Appelle und Angebote hinauszugehen, und diverse Anreizund Kontrollmechanismen zum Einsatz bringt. Bonusprogramme und Wahltarife der Krankenkassen stellen mit Sicherheit erst den Anfang einer Entwicklung dar, die Eigenverantwortlichkeit ins Zentrum eines zunehmend rudimentären Solidarsystems stellt. Dabei führen epidemiologische wie volkswirtschaftliche Logik dazu, dass die Grenzwerte, die die Grundlage der Risikoprofile darstellen, tendenziell immer weiter gesenkt werden, d. h. dass immer mehr Menschen als at risk klassifiziert werden. Eine aktuelle Studie im British Medical Journal zeigt, dass etablierte Parameter zur Diagnose des metabolischen Syndroms derzeit etwa $90 \%$ der über 50-jährigen Bevölkerung Norwegens einschließen (Westin/Heath 2005).

\footnotetext{
${ }^{5}$ Innerhalb epistemischer Kulturen (Knorr-Cetina 1999) wurde diese Form der Routinisierung bereits mit dem Konzept der epistemischen Objekts thematisiert (Rheinberger 1997).

${ }^{6}$ Siehe beispielhaft auch den Beitrag von S. Duttweiler in diesem Band.
} 
Der kanadische Wissenschaftshistoriker und -philosoph Ian Hacking spekulierte in seinem British Academy Vortrag des Jahres 2006, dass Übergewicht nicht „a way to be a person" sei und sich damit von schwerwiegenden Erkrankungen und Diagnosen wie Autismus oder Schizophrenie unterscheide (Hacking 2006). Für Übergewicht als einen isolierten physiologischen Zustand mag dies zutreffen. Risikoprofile bzw. Grenzwerte können allerdings in diesem Kontext nicht allein als objektive Parameter betrachtet werden. Sie stellen zum einen einen heftig umkämpften Konsens divergierender Expertenmeinungen innerhalb verschiedener wissenschaftlicher Disziplinen dar (Aronowitz 1998). Zum anderen spiegeln sie (volks)wirtschaftliche und politische Interessen wider. Als solche verknüpfen sie eine überwiegend medizinisch konnotierte Diagnose mit einem politisch-moralischen Diskurs, der Eigenverantwortlichkeit im Namen von Solidarität einfordert und Schuld zuschreibt.

Diese Form des Übergewichts als komplexe präventive Praxis kann in der Tat als „a way to be a person" verstanden werden, der in bestimmten Kontexten die Betroffenen zu einer Reaktion herausfordert. Dabei sind keinesfalls nur Reaktionen zu beobachten, die man als Technologien des Selbst in einem Diskurs der Biomacht verstehen könnte. Foucault hat zwar gezeigt, wie sich im Zuge der Moderne Regierungsformen etablieren, die ihre Wirkmächtigkeit und Stabilität darüber entfalten, dass sie Subjekte hervorbringen, die sich als Subjekte so konstituieren, dass sie dominante Diskurse und Herrschaftsverhältnisse reproduzieren (Foucault 1979). Dabei legt er allerdings weniger Wert auf eine Beschreibung und Analyse der Widerständigkeit von Subjekten und sozialen Konstellationen.

So entstand bereits 1969 die National Association for the Advancement of Fat Acceptan$c e$ (NAAFA) als eine Selbsthilfe- und Lobbygruppe. Neben dieser etablierten Institution entstehen derzeit zahlreiche size acceptance-Bewegungen vor allem in Nordamerika, aber zunehmend auch in Europa und Asien.77 Ziel dieser Gruppierungen ist es, ihre Mitglieder im Kampf gegen Diskriminierungen aller Art zu unterstützen. Dazu gehört zum einen die gegenseitige Unterstützung im dem Bestreben, Dicksein als positive Identität zu etablieren - fat is a neutral descriptor. Internetforen, Selbstberichte und Informationsbroschüren spielen dabei eine wichtige Rolle. Zum anderen treten gerade die größeren, gut organisierten Gruppen als Lobbyisten und Repräsentanten der Gemeinschaft der Übergewichtigen in politischen Anhörungen und Gerichtsverfahren auf (Saguy/Riley 2005).

Dabei ist vor allem ihre Auswertung und Nutzung aktueller medizinischer Forschungsergebnisse von Interesse. Von MedizinerInnen hört man häufig die Sorge, dass Berichte in den Medien über Dickmacher-Gene viele Übergewichtige dazu bewegen könnten, ihre Versuche, Gewicht zu verlieren, mit der Begründung aufzugeben, dass man gegen seine genetische Disposition nun einmal nichts machen könne. Und in der Tat taucht diese verkürzte Argumentation hier und da in dieser Reinform auf. Viel prominenter hingegen ist eine komplexere Variante dieses Themas, die auch auf wissenschaftlicher Seite Unterstützung findet. Es mache keinen Sinn sich gegen seine biologische Ausstattung zu wehren und Übergewicht sei auf vielfache Weise biologisch determiniert. Jedoch ließe sich trotzdem gegen Herzkreislauferkrankungen vorgehen. Diese Argumentation zeichnet das

\footnotetext{
${ }^{7}$ Siehe http://www.size-acceptance.org/branches/index.html für einen Überblick.
} 
Bild vom fitten Übergewichtigen, der zwar zuviel auf die Waage bringt, aber trotzdem nicht früher an Herzkreislauferkrankungen leidet als „Normalgewichtige“. Diese Position verschließt sich keineswegs der medizinischen Forschung, sondern kritisiert zum einen ihre Nähe zur Pharmaindustrie und die damit in Verbindung stehende Suche nach medikamentösen Lösungen. Zum anderen wehrt sie sich massiv gegen Diäten als Lösung für Probleme von Übergewichtigen. Auch hier spielt zum einen eine Industriekritik eine Rolle, zum anderen werden zahlreiche epidemiologische Studien aus verschiedenen Gründen als wenig aussagekräftig kritisiert ${ }^{8}$ Wichtiger ist allerdings die wenig strittige Beobachtung, dass die überwiegende Zahl der Diäten das Gewicht nicht verringert, sondern erhöht. Der Lösungsansatz Gewichtsverlust erhöhe damit das Risiko einer Herzkreislauferkrankung. Diese Argumentation kritisiert also vor allem die Interventionslogiken wie -praxen als weder theoretisch noch praktisch zu rechtfertigen. Sie nutzt damit eine Entkräftung der medizinischen Logik, um die Akzeptanz von Übergewicht als gleichberechtigter Lebensform zu befördern.

Saguy trennt in ihrer Analyse der konkurrierenden Rahmungen antiobesity ForscherInnen und AktivistInnen einerseits von fat acceptance ForscherInnen und AktivistInnen andererseits (Saguy/Riley 2005). Was ihre detaillierte Arbeit aber vor allem auch zeigt, ist die mannigfaltige Verschränkung von Wissenspraxen. Eine klare Trennung von wissenschaftlicher Praxis und politischer Arbeit lässt sich nicht aufrechterhalten, auch wenn Saguy dies versucht. Glaubwürdigkeit, Priorisierungen, persönliche wie politische Interessen und Ziele verschmelzen mit wissenschaftlicher Arbeit. Es wird zum einen deutlich, dass die Reduktion von Kontingenz in translationen selten vollständig gelingt. Hatte die Aneignung der ethnischen Stratifzierungskriterien noch den Erfolg solcher Praktiken gezeigt, betont diese Fallstudie die Widerständigkeiten. Zum anderen zeigt sich hier das von Hacking als looping beschriebene Phänomen (Hacking 1999). Die made up people, nämlich die als übergewichtig und Risikogruppe Klassifizierten, verhalten sich zu dieser Klassifizierung. Diese Widerständigkeit wirkt dann in die wissenschaftliche Praxis zurück und verändert dort die Diskussion über die Klassifizierungskriterien.

\section{Diskussion: Biopolitik und Subjektivierung}

Die Fallstudien verdeutlichen die Vielfältigkeit von translationen. Sie belegen, dass $m a-$ king up people selten lediglich einen linearen Prozess des Transfers und der Aneignung beschreibt, sondern durch eine Verschränkung bzw. durch die intersections von Wissenspraxen und Materialitäten gekennzeichnet ist, durch Rückkopplungseffekte und durch Diskontinuitäten. Der Fokus auf das Prozesshafte von translationen ermöglicht einen Blick hinter die Kulissen von scheinbar faktischem Wissen. Dabei geht es nicht um eine Entlarvung einer wissenschaftlichen Tatsache als soziale Konstruktion, denn ein solcher Ansatz setzt die notwendige Verknüpftheit von sozialer Praxis und Materialität voraus. Vielmehr macht diese Perspektive die Produktionsprozesse sichtbar, die an der Herstellung und Stabilisierung von Wissensbeständen beteiligt sind.

\footnotetext{
${ }^{8}$ Grundlage für diese Kritik sind vor allem Einschlusskriterien und das Fehlen valider Kontrollgruppen.
} 
Es geht allerdings nicht nur um die Details der Mechanik von Wissensproduktion. Translationen, so wie die Analyse der Fallstudien sie zeigt, formen einen wichtigen Bestandteil dessen, was Rabinow als new types of autoproduction und als Triebkraft von Biosozialität gekennzeichnet hat. Als solche können sie nicht isoliert betrachtet werden, sondern müssen als Bestandteil eines panoply of pastoral keepers in einen zunehmend transnationalen und neoliberalen Diskurs über Biopolitik und Biomacht verstanden werden. Rose und Rabinow haben mit Foucault zu Recht darauf hingewiesen, dass sich in der westlichen Welt zunehmend eine Biopolitik etabliert, die Strategien des Regierens über eine Beziehung von letting die (laissez mourir) und making live (faire vivre) entwickelt (Rabinow/Rose 2006). Die Entstehung von Präventionsgefügen, d. h. der Kampf gegen Übergewicht durch die Verschränkung verschiedenster Wissenspraxen, kann also in eine Entwicklung seit dem frühen 18.Jahrhundert eingeordnet werden, die der Wirkmächtigkeit von zunehmend biologisch geprägten Wahrheitsdiskursen und den damit verbundenen Akteuren und Praxisformen eine zentrale Rolle einräumt. Die neue Sprache der Suszeptibilität fungiert hier als integratives Moment (Rabinow/Rose 2006:3).

\section{Somatisierungsprozesse}

Im Rahmen dieser biopolitischen Überlegungen sind Subjektivierungsprozesse eingehend analysiert worden (Rose 1989; 1998). Dabei lässt sich eine Verschiebung ausmachen, die Körper und Körperlichkeit als Elemente subjektivierender Vektoren in den Vordergrund rückt. So sprechen Novas und Rose von „somatischer Individualität“, um zu kennzeichnen, dass Soma in verschiedenen Formen und auf verschiedenen Pfaden zunehmend in die Konstituierungsprozesse des Selbst impliziert ist: „recent developments in the lifesciences, biomedicine and biotechnology are associated with a general, somaticization " of personhood in an array of practices and styles of thought [...]" (Novas/Rose 2000: 491)

Problematischer gestaltet sich eine Analyse der kollektiven Dimension dieser Entwicklungen. Dabei ist zu beachten, dass in diesen Zusammenhängen häufig zum weiten Feld der genetischen Erkrankungen gearbeitet wird und es sich hier anbietet, Kollektivierung auf der Ebene von Population oder Familie zu denken (Armstrong et al 1998; Beck 2002; Strathern 1992). Dieser Fokus hat selbstverständlich seine Berechtigung, denn monogenetische Krankheiten machen Verwandtschaftsverhältnisse auf eine neue Art und Weise sichtbar und problematisieren sie mit einer bisher unbekannten Spezifik. Hinzukommt, dass die Technologien und Methoden, die zum Einsatz kommen, in hohem Maße öffentlich kontrovers diskutiert werden, hier also auch ein gesellschaftlicher Selbstverständigungsprozess über grundsätzliche Fragen von Leben, Krankheit und Tod beobachtbar wird. Gleichzeitig muss festgehalten werden, dass monogenetische Krankheiten sowohl für die Betroffenen als auch für die Gesellschaft insofern Ausnahmeerscheinungen darstellen, als sie wenige Menschen betreffen, in deren Alltag aber außerordentlich massiv eingreifen. Auch Hackings Konzepte des making up people und des looping funktionieren, wenn nicht notwendiger Weise mit genetischen, so doch mit schwerwiegenden Krankheiten, die Alltag radikal transformieren.

Die präventiven translationen im Kontext von Übergewicht vollziehen sich dagegen innerhalb bestehender Gewebe von Alltagspraxis. Kleine Verschiebungen, die isoliert 
eher banal erscheinen, die aber in ihrer additiven Gesamtheit eine durchaus gewichtige Positionsänderung ausmachen, entziehen sich rasch der analytischen Aufmerksamkeit. Ferner fehlt diesen graduellen Prozessen jeglicher Kristallisationspunkt, an dem sich Widerstand formieren könnte. Es ist daher wichtig hervorzuheben, dass die Beobachtungen und Befunde aus dem Bereich der schwerwiegenden genetischen Erkrankungen nur bedingt bei der hier vorliegenden Analyse Anwendung finden können.

So lässt sich sicherlich aufgrund der Fallstudien zu Ethnizität und fat acceptance analog zu Novas und Rose von somatischer Sozialität sprechen. Im Falle der National Association for the Advancement of Colored People wird eine spezielle Form der Körperlichkeit, Übergewicht, zu einem weiteren definierenden Merkmal von Gemeinsamkeit. Es ist fast schon ironisch, dass hier eine symbolisch-politische $\mathrm{Zu}$ (sammen)gehörigkeit plötzlich einen physiologischen Status verleiht. Im Gegensatz zu dieser Verschiebung, ist der Widerstand der fat acceptance Organisationen speziell auf die symbolische Politik des Übergewichts ausgerichtet. Körperlichkeit und Körperkonzepte sind hier explizites Programm, so dass man den Aufwind, den diese Bewegungen derzeit erfahren, als eine Facette einer Somatisierung von Sozialität verstehen kann.

Ob Widerständigkeit gegen oder Kooperation mit Klassifizierungsmechanismen: Kollektivierungsdynamiken inkorporieren zunehmend eine somatische Komponente. Dabei tritt Soma allerdings nicht nur als kollektivierender Vektor im Sinne somatischer Kollektivierungsdynamiken auf. Hackings looping weist daraufhin, dass diese Entwicklungen immer auch somatische Auswirkungen haben. Man muss also ebenfalls von kollektiven Somatisierungsdynamiken sprechen, um nicht aus dem Blick zu verlieren, dass diese Formen der institutionellen Kollektivierung immer auch Körper im Sinne von Physiologie verändern. Da dieser Prozess vermittelt auftritt, ist es eine zentrale Aufgabe aktueller Forschung, zum einen die entstehenden Interventionen, Subventionen und Sanktionen zu analysieren. Zum anderen wird es interessant sein zu beobachten, ob und wie Konzepte von Verantwortung, Bildung und Gemeinschaft eine somatische Umdeutung erfahren (Beck/Niewöhner 2006).

\section{Was bleibt von den Fetten?}

So entfaltet und verschränkt sich im Falle von Prävention und Übergewicht in der Tat ein panoply of pastoral keepers, das Kollektivierungsdynamiken formt und somatisiert. Rabinow hatte im ursprünglichen Entwurf von Biosozialität das Konzept nicht unironisch überzeichnet, um soziobiologische Entwicklungen zu karikieren (Rabinow 1992). Die subtilere Variante von Biosozialität im Kontext aktueller Überlegungen zu Biomacht (Rabinow/Rose 2006) zielt auf Formen der sozialen und individuellen Transformation, die durch Regierungsformen bestimmt werden, die life itself (Rose 2001) zum Ziel haben und sich zum einen über Technologien des Selbst manifestieren. Zum anderen verändern sie Kollektivierungsdynamiken und Sozialität über Klassifizierungspraxen, die auf genetischen, physiologischen oder ethnischen Kategorien aufbauen, ihre eigentliche Wirkmächtigkeit aber über eine Verschränkung mit politisch-moralischen wie (volks)wirtschaftlichen Kategorien entfalten. 
Der kürzlich im Deutschen Bundestag vorgestellte nationale Aktionsplan „Fit statt Fett" des Bundesministeriums für Ernährung, Landwirtschaft und Verbraucherschutz zeigt deutlich, dass der Kreuzzug gegen die Fette noch eine lange Zukunft vor sich hat. Aufklärung, Eigenverantwortlichkeit und Lebensqualität sind z. Z. noch die dominanten Schlagworte, mit denen man eine "Olypmiade der Verbote" verhindern will. Aber die weniger subtilen Steuerungs- wie Sanktionsmechanismen, z. B. Wahltarife und Bonussysteme der Krankenkassen, sind bereits erarbeitet bzw. in Kraft.

Die Logik dieses Gefüges zu beobachten und zu analysieren wird daher eine wichtige Aufgabe der Forschung der nächsten Jahre darstellen. An der Schnittstelle von Wissenschaftsforschung, Sozialanthropologie und Lebenswissenschaften werden dabei vor allem die Verschränkungen von Expertensystemen und sozialem Alltag in den Vordergrund rücken, die so häufig nur als nicht intendierte Nebenfolgen thematisiert werden. Die Beobachtung und Analyse dieser Entwicklungen muss dabei zwei Dinge im Blick halten können: Erstens haben widerständige wie konforme Formen von somatischer Sozialität immer auch somatische Konsequenzen, d. h. sie verändern Körper als Materialität und nicht nur auf politisch-symbolischer Ebene als Text und Diskurs. Governance at a distance hält also nicht beim Subjekt an, sondern greift auf das Molekulare durch. Dies erfordert Forschungsdesigns, die die Brücke zwischen Molekularem und Sozialem erfassen und bearbeiten können und sich nicht schon qua Methode auf bestimmte Analyseebenen festlegen müssen (Beck/Niewöhner 2006). Zweitens stellt sich die Frage, wie ein langsamer Verschiebungsprozess, der unspektakulär in das Gewebe eines ausdifferenzierten Alltags verwoben ist, im Jetzt registriert werden kann. Der Versuch einer anthropology of the contemporary 9 hat hier einen wichtigen Anfang gemacht. Dabei könnte die Entwicklung einer unnachgiebig relationalen Perspektive (Gane/Haraway 2006) dazu beitragen, scheinbar banale Verschiebungen in Netzwerken anders zu registrieren als dies mit etablierten akteurszentrierten Perspektiven der Fall wäre.

\section{Literatur}

AAA. (1998): Statement on 'Race'. http://www.aaanet.org/stmts/racepp.htm, letzter Zugriff 29.03.2007, American Anthropological Association, Arlington, VA.

Armitage, J.A./Khan, I.Y./Taylor, P.D./Nathanielsz, P.W./Poston, L. (2004): Developmental programming of the metabolic syndrome by maternal nutritional imbalance: how strong is the evidence from experimental models in mammals? In: Journal of PhysiologyLondon 561, S. 355-77.

Armstrong, D./Michie, S./Marteau, T. (1998): Revealed Identity: a study of the process of genetic counselling. In: Social Science \& Medicine 47, S. 1653-8.

Aronowitz, R. (1998): Making sense of illness. Science, Society and Disease. Cambridge. Beck, S. (2002): Frozen altruism, heated solidarity and iced ethics. In: Biobanks and

$\sqrt[9]{\text { http://anthropos-lab.net/ }}$ 
Ethics, Lundin (Hrsg.).

Beck, S./Niewöhner, J. (2006): Somatographic investigations across levels of complexity. In: Journal of BioSocieties 1, S. 219-27.

Bijker, W.E./Hughes, T.P./Pinch, T.P., eds. (1987): The Social Construction of Technological Systems. New Directions in the Sociology and History of Technology. Cambridge.

Bloor, D. (1991): Knowledge and Social Imagery. Chicago.

Brunner, E.J./Hemingway, H./Walker, B.R./Page, M./Clarke, P., et al. (2002): Adrenocortical, autonomic, and inflammatory causes of the metabolic syndrome - Nested casecontrol study. In: Circulation 106, S. 2659-65.

Callon, M. (1999): Some Elements of a sociology of translation. Domestication of the Scallops and the Fishermen of St.Brieuc Bay. In The Science Studies Reader, Biagioli, M., New (Hrsg.) York, S. 67-84.

Cheal, K.L./Abbasi, F./Lamendola, C./McLaughlin, T./Reaven, G.M./Ford, E.S. (2004): Relationship to insulin resistance of the Adult Treatment Panel III diagnostic criteria for identification of the metabolic syndrome. In: Diabetes 53, S. 1195-200.

Duster, T. (2006): Lessons from History: Why Race and Ethnicity have played a major role in biomedical research. In: Journal of Law, Medicine \& Ethics Fall 2006, S. 1-11.

Foucault, M. (1979): The history of sexuality - Vol I.: An introduction. London.

Fujimura, J.H. (1992); Crafting science: standardized packages, boundary objects, and „translation“. In Science as Practice and Culture, ed. Pickering, A. Chicago.

Gane, N./Haraway, D. (2006): When we have never been modern, what is to be done. In: Theory, Culture \& Society 23, S. 135-58.

Hacking, I. (1999): The social construction of what? Cambridge/MA.

Hacking, I. (2006): Kinds of People: Moving Targets. In: British Academy Lecture 10, S. 1- 18.

Hedgecoe, A. (2004): The politics of personalised medicine: pharmacogenetics in the clinic. Cambridge.

IDF (2006): The IDF worldwide consensus definition of the metabolic syndrome, International Diabetes Foundation, Brussels.

Irwin, A. (2001): Constructing the scientific citizen: science and democracy in the biosciences. In: Public Understanding of Science 10, S. 1-18.

Joerges, B. (1987): Technik, Umwelt, Alltag - eine Bestandsaufnahme neuerer soziologischer Forschung. Berlin: Wissenschaftszentrum.

Joerges, B. (1996): Technik, Körper der Gesellschaft. Frankfurt/M.

Kahn, R./Buse, J./Ferrannini, E./Stern, M. (2005): The metabolic syndrome - time for a critical appraisal. In: The Lancet 366, S. 1921-2. 
Knorr-Cetina, K. (1999): Epistemic Cultures. Cambridge/MA.

Kollek, R./Feuerstein, G./Schmedders, M./Aken, J.v. (2003): Pharmakogenetik: Implikationen für Patienten und Gesundheitswesen. Baden-Baden.

Latour, B. (1995): Wir sind nie modern gewesen. Versuch einer symmetrischen Anthropologie. Berlin.

Latour, B. (2005): Reassembling the Social: An Introduction to Actor-Network-Theory. Oxford.

Law, J. (1992): Notes on the Theory of the Actor Network: Ordering, Strategy, and Heterogeneity. In Working Paper Series Lancaster University CSS. Lancaster.

Law, J./Hassard, J., eds. (1999): Actor Network Theory and After. Oxford: Blackwell and the Sociological Review.

Lipphardt, V./Niewöhner, J. (2007): Producing difference in an age of biosociality. Biohistorical narratives, standardisation and resistance as translations. In: Science, Technology \& Innovation Studies 3, S. 45-66.

Lock, M./Young, A./Cambrosio, A., eds. (2000):. Living and Working with the New Medical Technologies. Intersections of Inquiry. Cambridge.

NCEP (2001): Executive summary of the third report of the national cholesterol education program (NCEP) expert panel on detection, evaluation, and treatment of high blood cholesterol in adults (adult treatment panel III). In: Journal of the American Medical Association 285, S. 2486-97.

Novas, C./Rose, N. (2000): Genetic risk and the birth of the somatic individual. In: Economy and Society 29, S. 485-513.

Pasanisi, P./Berrino, F./De Petris, M./Venturelli, E./Mastroianni, A./Panico, S. (2006): Metabolic syndrome as a prognostic factor for breast cancer recurrences. In: Int J Cancer.

Rabinow, P. (1992): From sociobiology to biosociality: artificiality and enlightenment. Incoroporations. Crary, J./ Kwinter, S. (Hrsg.). New York.

Rabinow, P./Rose, N. (2006): Biopower today. In: Journal of BioSocieties, :S. 195-217.

Reaven, G. 1988. Banting lecture (1988): Role of insulin resistance in human disease. In: Diabetes 37, S. 1595-607.

Reaven, G. (2002): Metabolic syndrome - Pathophysiology and implications for management of cardiovascular disease. In: Circulation 106, S. 286-8.

Rheinberger, H.-J. (1997): Toward a History of Epistemic Things. Synthesizing Proteins in the Test Tube. Stanford.

Rose, N. (1989): Governing the Soul - the shaping of the private self. London: Routledge.

Rose, N. (1998): Inventing Our Selves - Psychology, Power and Personhood. Cambridge.

Rose, N. (2001): The Politics of Life Itself. In: Theory, Culture \& Society 18, S. 1-30. 
Rosmond, R./Bjorntorp, P. (2000): Occupational Status, Cortisol Secretory Pattern, and Visceral Obesity in Middle-aged Men. In: Obes Res 8, S. 445-50.

Saguy, A.C./Riley, K.W. (2005): Weighing both sides: Morality, Mortality, and Framing Contests over Obesity. In: Journal of Health Politics, Policy and Law 30, S. 869-921.

Stifterverband (2000): PUSH - Public understanding of science and humanities, Stifterverband für die deutsche Wissenschaft, Bonn.

Strathern, M. (1992): After Nature: English Kinship in the Late twentieth Century. Cambridge.

Tauman, R./O'Brien, L.M./Ivanenko, A./Gozal, D. (2005): Obesity Rather Than Severity of Sleep-Disordered Breathing as the Major Determinant of Insulin Resistance and Altered Lipidemia in Snoring Children. In: Pediatrics 116, S. 66-73.

Walter, U./Scriba, P.C. (2004): Präventive Medizin: Sind wir noch nicht reif? In: Der Internist 45, S. 137-8.

Westin, S./Heath, I. (2005): Thresholds for normal blood pressure and serum cholesterol. In: BMJ 330, S. 1461-2.

WHO Expert Consultation (2004): Appropriate body-mass index for Asian populations and its implications for policy and intervention strategies. In: The Lancet 363. S. 159-63.

Wynne, B. (1996): Misunderstood misunderstandings: social identities and public uptake of science. In Misunderstanding science, Irwin, A./ Wynne, B. (Hrsg.). Cambridge.

Zimmet, P.Z./Alberti, K.G.M.M./Shaw, J.E. (2005): Mainstreaming the metabolic syndrome: a definitive definition. In: Medical Journal Australia 183, S. 175-6. 